\title{
Readout electronics for low dark count pixel detectors based on Geiger mode avalanche photodiodes fabricated in conventional CMOS technologies for future linear colliders
}

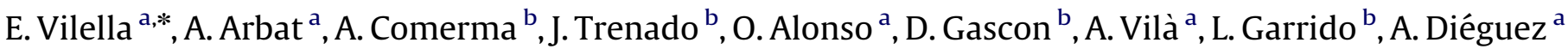 \\ a Department of Electronics, University of Barcelona (UB), Martí i Franquès 1, 08028 Barcelona, Spain \\ ${ }^{\mathrm{b}}$ Department of Structure and Constituents of Matter, University of Barcelona (UB), Martí i Franquès 1, 08028 Barcelona, Spain
}

\section{A R T I C L E I N F O}

\section{Available online 21 December 2010}

\section{Keywords:}

High energy physics experiments

Avalanche photodiodes

Dark count rate

Forward tracking detector

Low overvoltage

Pixel

Technology comparison.

\begin{abstract}
A B S T R A C T
High sensitivity and excellent timing accuracy of the Geiger mode avalanche photodiodes make them ideal sensors as pixel detectors for particle tracking in high energy physics experiments to be performed in future linear colliders. Nevertheless, it is well known that these sensors suffer from dark counts and afterpulsing noise, which induce false hits (indistinguishable from event detection) as well as an increase in the necessary area of the readout system. In this work, we present a comparison between APDs fabricated in a high voltage $0.35 \mu \mathrm{m}$ and a high integration $0.13 \mu \mathrm{m}$ commercially available CMOS technologies that has been performed to determine which of them best fits the particle collider requirements. In addition, a readout circuit that allows low noise operation is introduced. Experimental characterization of the proposed pixel is also presented in this work.
\end{abstract}

(c) 2010 Elsevier B.V. All rights reserved.

\section{Introduction}

High energy physics experiments aimed to study the smallest constituents of matter and the fundamental interactions between them are performed at particle colliders. At present time, there exist a wide number of particle colliders all around the world, such as the Large Hadron Collider (LHC) in Geneva or the KEKB electronpositron collider in Tsukuba (Japan). However, most of the current colliders are circular, which induce energy losses in the accelerated beam that limits the beam energy. This drawback has motivated the development of linear colliders, such as the future International Linear Collider (ILC).

In order to achieve the purpose of the experiment, in future linear colliders there are various types of detectors, each of which performs one specific function. Thereby, the forward tracking detector in ILC, which is expected to be composed of seven disks based on silicon technology, measures the curvature of the trajectory of charged particles in order to reconstruct their momentum. To successfully achieve this goal, detector technology with excellent performance is a must. In particular, sensors have to fulfill a set of specific requirements that were defined in Ref. [1]. First, sensitive elements with high granularity for high spatial resolution as well as low material budget $\left(<0.1 \% X_{0}\right.$ per layer) to reduce the error from multiple scattering are needed for efficient

\footnotetext{
* Corresponding author. Tel.: +3493 40391 46; fax.: +3493402 1148 .

E-mail address: evilella@el.ub.es (E. Vilella).
}

measurements. Second, it is widely accepted that the size of the sensors has to be around $20 \mu \mathrm{m} \times 100 \mu \mathrm{m}$ to provide adequate spatial resolution for the reconstruction of the tracks (i.e., to determine the production point of a new particle with an error smaller than $5 \mu \mathrm{m}$ ) and also to reduce the occupancy of the detector. Moreover, the readout speed must be fast to meet the constraints of the experiment. Apart from this, another serious specification imposed by the collider environment is related to radiation tolerance and electromagnetic interferences (EMIs). Finally, minimum power dissipation is compulsory due to no plan for cooling system.

There is now unanimity regarding the basic detector technology for ILC. Silicon pixel detectors (either hybrids or monolithics) and silicon microstrips are accepted as the options for the three inner layers and for the four outer layers of the forward tracker detector, respectively. However, the sensor technology of the silicon pixel detector has not yet been decided. Consequently, a large variety of sensor candidates has blossomed recently in an attempt to fulfill the specifications posed by the collider. Sensitive elements such as CCDs [2] and MAPS [3], as well as their derivatives, and more recently DEPFETs [4] can be found in the current state of the art [5]. The sensors are connected to the readout electronic circuits, which are preferably integrated on the same substrate. Although all the present options have a chance of achieving the goal, none of them is guaranteed to satisfy all the specifications. More recently, a very interesting alternative based on 3D integration [6] has appeared, but this option is at an early stage of development since costs of fabrication have been a serious drawback. 
In addition, Geiger mode Avalanche PhotoDiodes (GAPDs) are introduced as one attractive candidate for tracking systems [7] due to their high sensitivity and excellent timing accuracy. Indeed, they enable single hit detection within ps rise times and short recovery periods. GAPDs, however, suffer from dark counts and afterpulsing pulses, which are impossible to distinguish from real counts. Moreover, this phenomenon represents a severe limitation of the photodiode and also an increase in the necessary area of the readout electronics to store the false hits.

In this article, we present the results of a comparative study between APDs fabricated with two commercially available standard CMOS technologies (HV-AMS $0.35 \mu \mathrm{m}$ and STM $0.13 \mu \mathrm{m}$ ). We also propose fast enough readout electronic circuitry with the HV-AMS $0.35 \mu \mathrm{m}$ technology that replaces the typical CMOS comparator and allows low noise GAPD operation. The characterization of the proposed pixel is also described here.

\section{Experimental}

Different APD sensors and pixels have been fabricated in two CMOS processes in order to perform a comparative study. APD detectors with sensor size of AMS R1 CX $20 \times 20$ and AMS R2 CX $20 \times 20$ have been done in Austria Microsystems $0.35 \mu \mathrm{m}-\mathrm{HV}$ technology (process h35b4). The second run with the same technology was done to observe differences between runs. In this case, sensors of AMS R2 CX $20 \times 100$ were included, as well. Finally, another two runs with the $0.13 \mu \mathrm{m}$-STMicroelectronics process were also done (STM R1 CX $20 \times 20$ and STM R2 CX $20 \times 20$ ). For both technologies, we have characterized the $I(V)$ in the reverse mode, the dynamic behavior, the dark count rate, the afterpulsing probability and also the dependence with the temperature. Although the characterization of both technologies suggests at this moment that neither of them satisfies all the requirements imposed by the particle collider, we have chosen the HV-AMS $0.35 \mu \mathrm{m}$ technology for our GAPD pixel detector due to its lower dark count rate.

\section{Avalanche photodiodes}

Avalanche photodiodes are solid state sensor devices based on a $\mathrm{p}-\mathrm{n}$ junction that theoretically enables single particle detection when they are reverse biased in the so-called Geiger mode (i.e., above their breakdown voltage $V_{\mathrm{BD}}$ ). In this configuration, a high electric field is generated within the $\mathrm{p}-\mathrm{n}$ junction forming the multiplication region. Thus, if a sufficiently energetic ionizing particle traverses the depletion region, it generates an electronhole pair which, in turn, initiates an avalanche process of generation of electron-hole pairs in the high electric field region. As a result, the diode's depletion capacitance is rapidly discharged and a self-sustained current pulse, known as avalanche current, is generated. This current needs to be stopped in a short period of time in order to avoid burning of the device. This operation is performed by the quenching circuit [8], which lowers the reverse bias voltage of the sensor below its breakdown voltage. Quenching circuits can be implemented by means of passive or active components, although mixed solutions are also possible [9]. During the period of time in which the sensor remains under the breakdown voltage, known as dead time, detection of new particles is not allowed. Therefore, the sensor needs to recover its bias condition in order to enable new particle detection in the so-called recharge time. Thus, the dead time is the addition of the quenching and recharge times.

In addition, another mode of operation can be distinguished for APD sensors. In contrast to the Geiger mode, APDs are biased slightly below their $V_{\mathrm{BD}}$ work in the linear mode. In this case, when a charged particle traverses the $\mathrm{p}-\mathrm{n}$ junction, a proportional nonself-sustained electrical current is generated. However, the gain is low. Therefore, the linear mode is not recommended for particle tracking detectors since the detection of single particles is difficult.

A major issue of concern in GAPDs is the noise, which is dominated by dark counts and afterpulsing pulses. These noise sources cause false hits that cannot be distinguished from particle hits. Dark counts are spurious pulses due to thermally or tunneling induced carriers in the photodiode junction. The dark count rate depends on the technology, the sensitive area of the detector, the excess bias voltage and the temperature. On the other hand, afterpulses correspond to false avalanches due to carriers captured by traps in the multiplication region during an avalanche and released at a random time. The afterpulsing probability is a function of the number of carriers involved in an avalanche, which in turn depends on the GAPD parasitic capacitance. The afterpulsing probability can be reduced by increasing the dead time.

\section{Technologies under comparison and characterization of the sensors}

Two different CMOS standard technologies have been used to fabricate two similar detectors for comparison purpose. The first technology is the high voltage Austria Microsystems (HV-AMS) $0.35 \mu \mathrm{m}$ technology and the second one is the ST Microsystems (STM) $0.13 \mu \mathrm{m}$ technology. The HV-AMS technology offers a low doping deep n-well layer, which provides lower dark count rate. The STM technology offers the possibility to reduce the area occupied by the readout electronics and improve the fill factor. The structures under test are composed of a p-diffusion inside an n-well. To ensure a horizontal multiplication region and avoid the premature breakdown at the edges, a p-guard ring is implemented around the whole structure. Two chips containing different elements and including the presented structures for each technology have been fabricated and tested. The measurements shown here correspond to the four detectors. Specific remarks to the influence of process variations are given.

Using a Keithley 2611A source the current-voltage characteristic in the reverse bias mode of the detectors has been obtained (Fig. 1). The breakdown voltage of the STM technology is lower and

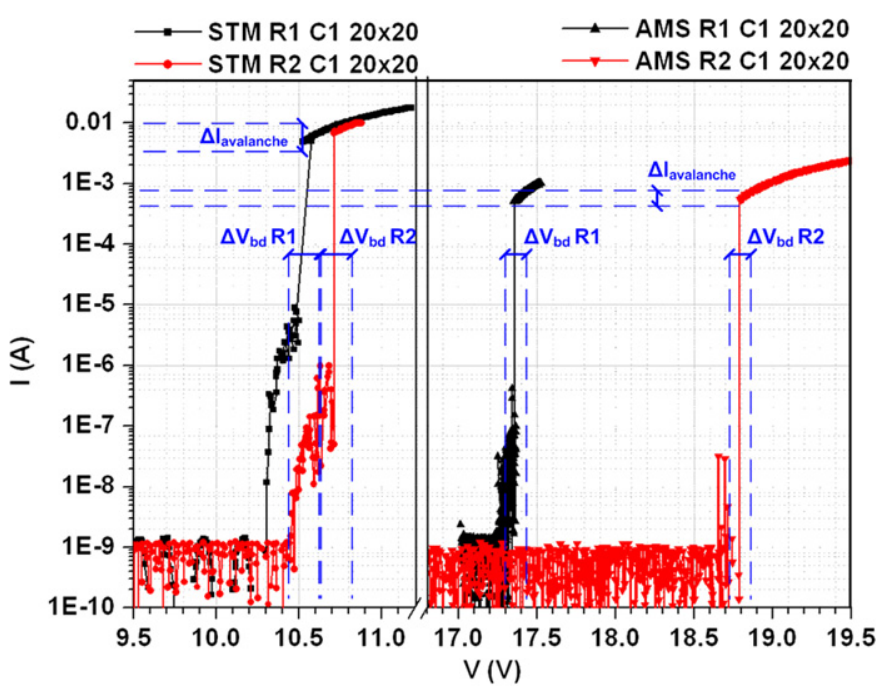

Fig. 1. Characteristics for detectors fabricated in different runs of the two technologies under comparison. Variation in the breakdown voltage observed between chips of the same run has been included. Avalanche current variation range has also been included for each technology. 
the variation between different dies is much lower than the one presented in the HV-AMS technology. Die to die variations (in the same run) give rise to hundred millivolts for the STM technology and $50 \mathrm{mV}$ for the HV-AMS technology. The breakdown voltage of the $\mathrm{p}-\mathrm{n}$ junction increases with temperature, which can be compensated by increasing the high voltage biasing of the detector to adjust the operating point of the electronic readout (Fig. 2).

A passive quenching composed of a $10 \mathrm{k} \Omega$ resistance is used to measure the noise and dynamic behavior of the sensor. The system is powered with an Agilent E3621A voltage source and the measurements are made using an FPGA and an oscilloscope TDS7154B with active probe. The dynamic response of the detectors using this setup is represented in Fig. 3. The quenching time of the detectors is related to the avalanche current generated and to the parasitic capacitors (detector capacitor, bonding pad and probes). Recovery time also depends on the parasitic capacitors, but it is mainly due to the capacitance of the detector. The HV-AMS technology is slower, as deduced from the higher recovery times. It is also clear that without embedding the electronic readout it is not possible to use these APDs for single bunch crossing detection in a tracker.

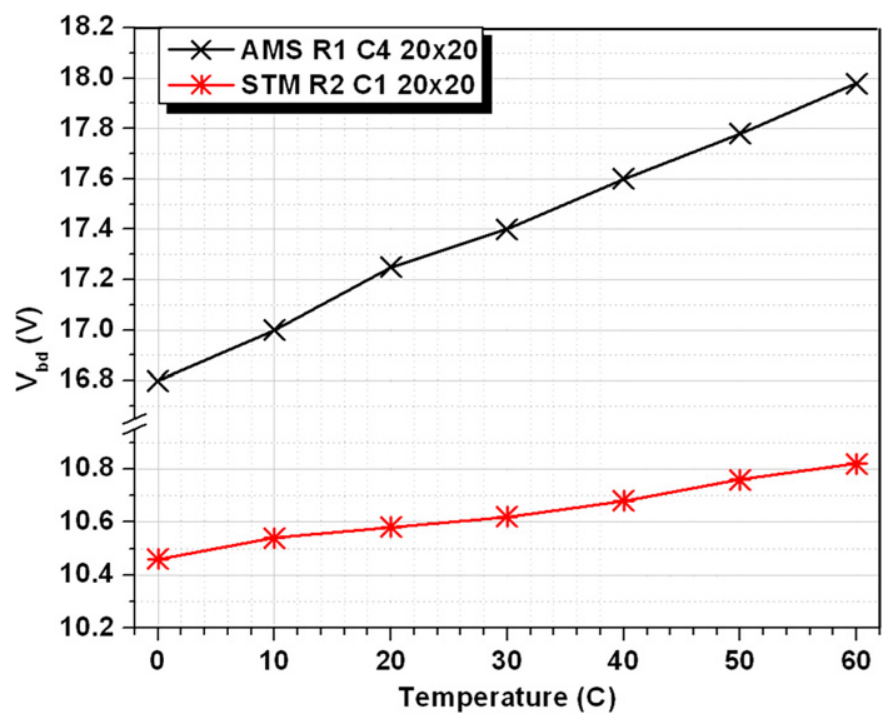

Fig. 2. Temperature dependence of the breakdown and dark count rate for both technologies.

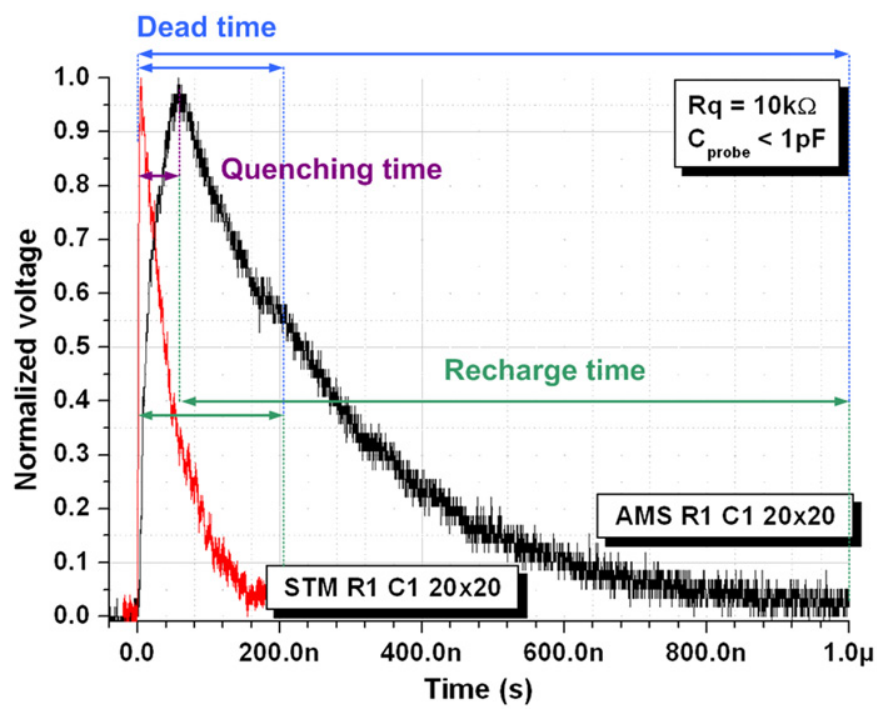

Fig. 3. Dynamic behavior of the detectors and timing definitions.
Dark count rate is obtained by making a statistical analysis on the number of pulses generated by the detector in a fixed window of time for different biasing voltages. The values measured for low overvoltages are represented in Fig. 4. Experimental results show a lower dark count rate for the HV-AMS technology than for the STM technology in all the investigated ranges. A higher variation in the dark count rate value than in the STM technology is observed. As pulses generated by dark count cannot be distinguished from pulses generated by particle crossing, all the hits generated during the detection mode have to be stored for post-processing. The higher value of dark count in the STM technology implies that a larger amount of false hits is produced and the probability of loosing real hits is larger. The value of the dark count increases with the increase in temperature, which worsens the signal-to-noise ratio of the detector (Fig. 2).

To observe the afterpulsing of the detector, the infinite permanence option of the oscilloscope is used. During a certain period of time, the signal is integrated in the oscilloscope screen where a pulse has been triggered. Afterpulsing generates a shadow of the triggered pulse, as shown in Fig. $5 \mathrm{~A}$, which corresponds to the HV-AMS technology. No afterpulsing is observed in Fig. 5B, corresponding to the STM technology. The afterpulsing probability for the HV-AMS technology is affected by the high quenching time observed in Fig. 3. When the electronic front-end is embedded in the chip, the capacitance of the sensing node is reduced and the quenching time too. This reduces the amount of charge flowing through the detector and thus the trapping probability. As the concentration of filled traps is lower, the afterpulsing probability is decreased.

The features of the detectors presented here make more suitable to use of the HV-AMS technology for the forward tracker than the STM technology. This decision has been taken mainly due to the dark count rate presented by both technologies, but also considering the variations in the breakdown voltage between dies, which are lower in the HV-AMS technology. Nevertheless, further analysis of the dark count with temperature and on irradiated sensors has to be performed to have concluding results.

\section{Readout electronics}

In optical systems based on GAPDs, the signal-to-noise ratio is obtained on counting detection events and comparing with the noise level. To cope with the dark count noise, it is necessary to design specific readout electronics coupled to the sensor with the intention to be insensitive as much as possible to dark counts.

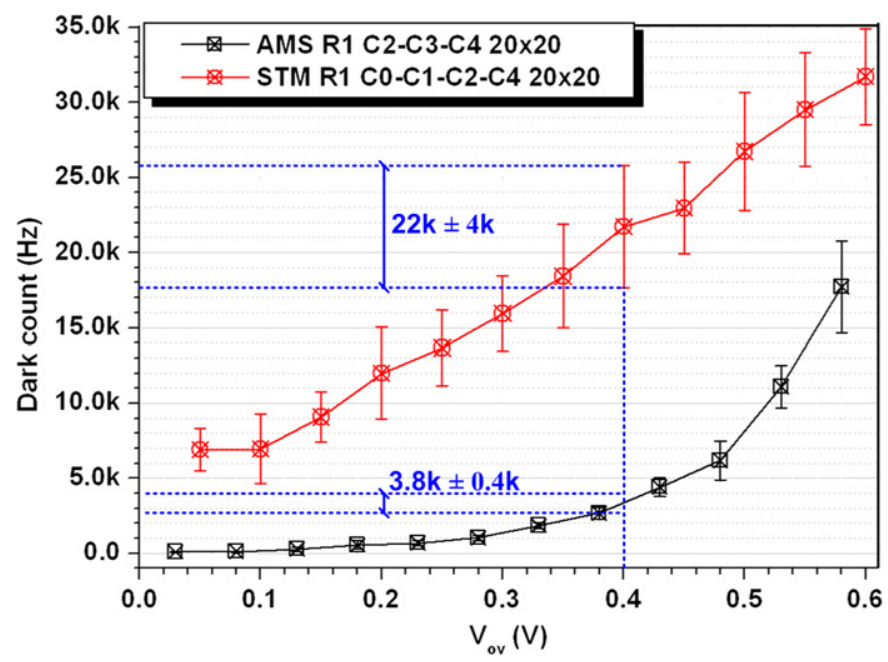

Fig. 4. Dark count rate obtained measuring different dies of the same run of the two technologies for low overvoltages. 

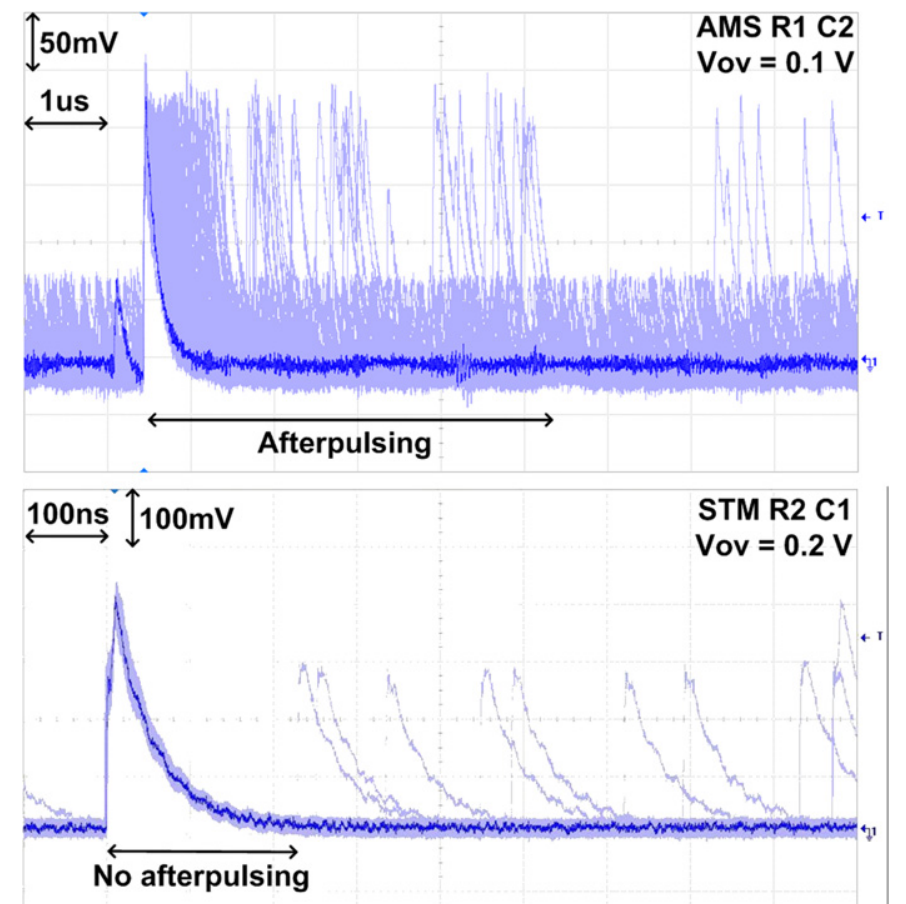

Fig. 5. Afterpulsing probability for each technology using external passive quenching.

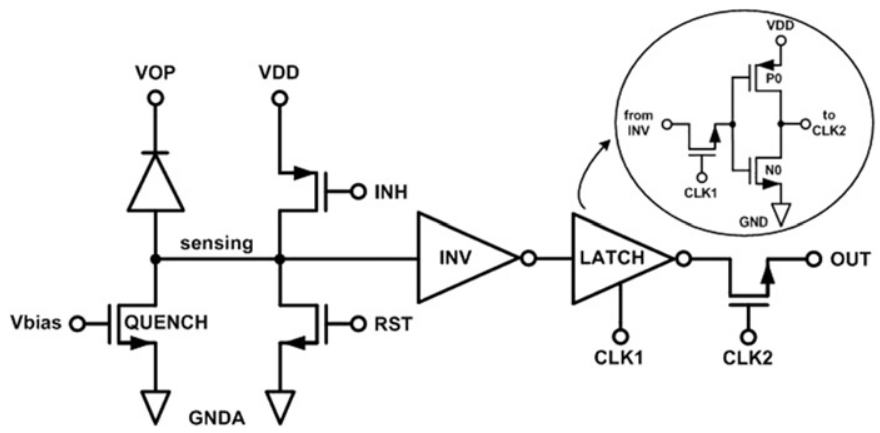

Fig. 6. Front-end circuit based on a floating scheme.

Fig. 6 shows the electronics designed to deal with noise in GAPDs for a tracker. The schematic diagram of the proposed pixel basically consists of an APD, a quenching transistor (QUENCH), an inverter for the avalanche event detection (INV), a dynamic latch to store the resultant value of the observation period (LATCH) and a pass gate to read out the pixel. To save area in an environment where the fill factor is a key parameter, the quenching block has been implemented by means of an active load based on an nMOS transistor with proper aspect ratio $(W / L)$. The quenching transistor is biased through a current mirror, which leads to superior performance to that provided by a MOS transistor operated as a resistor [10]. Three control signals have been introduced to enable the gated mode of operation, and consequently the synchronization with the beam timing structure. First, the dynamic latch, consisting of an nMOS pass gate and an inverter, is controlled by the CLK1 external signal. Second, a pMOS transistor (INH) connected between the power supply and the sensing node has been added to inhibit particle detection when necessary. Therefore, when the INH transistor is disabled and the CLK1 transistor is switched on, it occurs at the sensitive window of the detector. After the 'on' period, the INH transistor is switched on and the photodiode remains biased below $V_{\mathrm{BD}}$ during the preselected 'off' period. In the last place, before a new 'on' period is started, a reset transistor (RST) connected between the sensing node and GNDA is turned on so that it quickly recharges the GAPD by restoring its reverse bias voltage. In addition, and considering the future development of a $2 \mathrm{D}$ matrix detector of GAPD pixels, a second pass gate with another external gate control (CLK2) has also been included to read out the value of the pixel whenever it is desired.

As shown in Fig. 4, the dark count increases with the overvoltage. Although a wide range of readout configurations for GAPDs can be found in the literature [11], none of them is focused on low overvoltages to reduce the dark count noise. In our case, to operate the sensor with low overvoltages, the ground of the sensor (GNDA) is raised with regard to the ground of the detection and storage electronics (GND). Fig. 7 shows the waveforms corresponding to two complete cycles of the pixel detector. In the first case, there has been particle detection and the output node is set to logic ' 1 '. In contrast, during the second 'on' period no particle has been detected and the output node takes the value of logic ' 0 '.

The pixel detector, among another analog and digital blocks, was prototyped in HV-AMS $0.35 \mu \mathrm{m}$ technology. The microphotograph of the proposed pixel is shown in Fig. 8. The total area of the readout module is $40.6 \mu \mathrm{m} \times 10.8 \mu \mathrm{m}$. The detector was characterized through the test setup that can also be seen in Fig. 8 . The chip is arranged in a test board with discrete components. Moreover, to generate the three power supplies that are needed $\left(V_{\mathrm{OP}}\right.$, GNDA and $\left.V_{\mathrm{DD}}\right)$, an Agilent E3631A voltage source is used. Also, a FPGA has been used to generate the fast control signals.

In Fig. 9 the dark count measured with the described pixel is plotted. Enough statistics have been offered by measuring 200 times the windows of 50-300 ns for one single pixel. The measurement has been done for overvoltages of $0.5,1$ and $1.5 \mathrm{~V}$. The dark count has been calculated dividing the number of false counts by the window of observation. It is observed that the dark count rate is

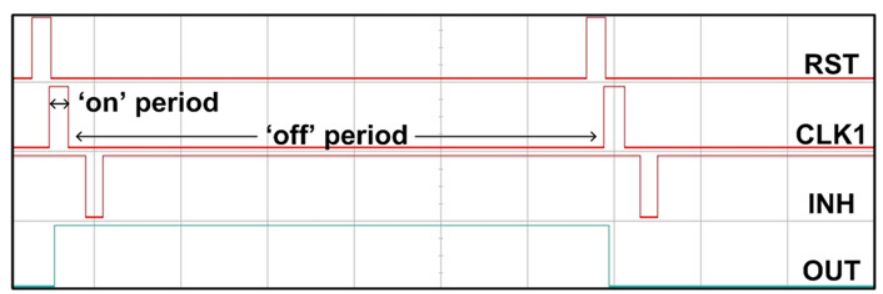

Fig. 7. Control and output waveforms.

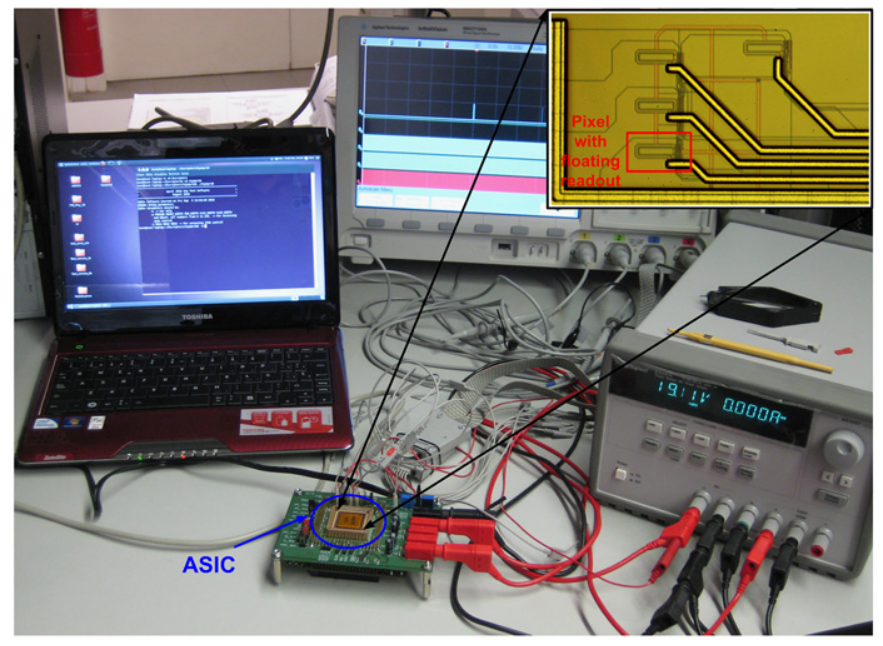

Fig. 8. Microphotograph of the proposed pixel and setup for the test characterization. 


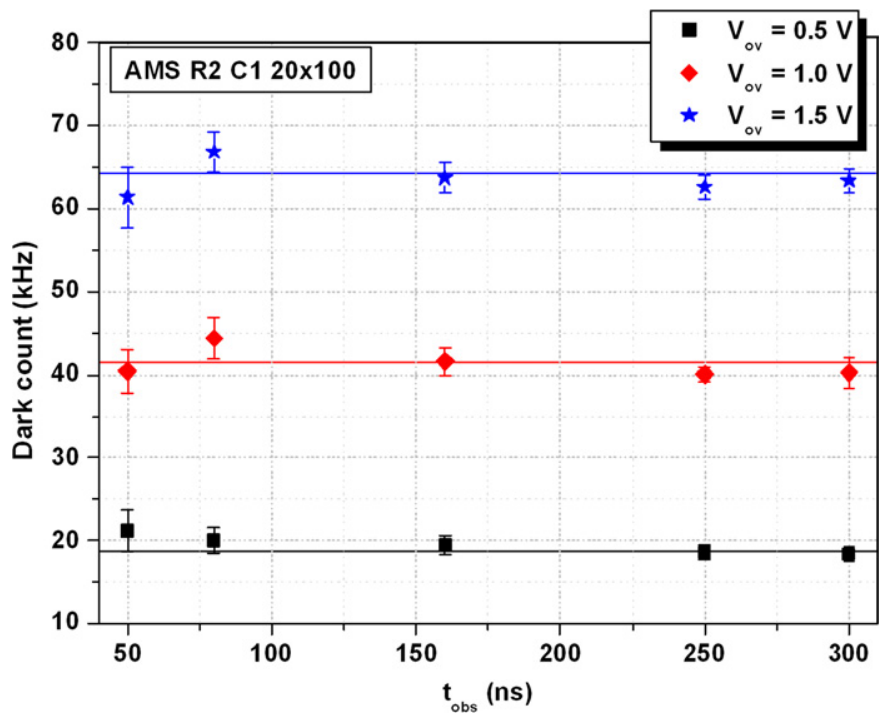

Fig. 9. Dark count rate of the proposed pixel for different reverse bias overvoltages.

Table 1

Performance of the detector.

\begin{tabular}{ll}
\hline Characteristics & Value \\
\hline Sensitive sensor area $(\mu \mathrm{m} \times \mu \mathrm{m})$ & $20 \times 100$ \\
Electronics area $(\mu \mathrm{m} \times \mu \mathrm{m})$ & $40.6 \times 10.8$ \\
$V_{\mathrm{BD}}(V)$ & 18.9 \\
Dark count at $V_{\mathrm{ex}}=0.5 \mathrm{~V}(\mathrm{kHz})$ & 19 \\
Afterpulsing & Not observed \\
Propagation delay of '0' (ns) & 1.8 \\
Propagation delay of ' 1 ' $(\mathrm{ns})$ & 0.5 \\
Current consumption $(\mathrm{pA})$ & 10 (hit)/4 (no hit) \\
\hline
\end{tabular}

Measured performance of the fabricated pixel detector.

constant for the window of observation, which confirms that the dark count is randomly generated and that the circuit does not affect the measurement. For a given window it is followed that $D C_{\mathrm{obs}}=$ $D C \cdot t_{\text {obs }}$. Therefore, reducing the window size the probability of obtaining a false hit due to the dark count is also reduced. Consequently, this is an effective method to reduce the noise in GAPD pixels for a tracker. The main characteristics of the fabricated pixel detector in the HV-AMS technology are summarized in Table 1.

\section{Conclusion}

Silicon pixel detectors based on GAPDs have been studied and characterized for tracking system applications. First, a comparative study between the HV-AMS $0.35 \mu \mathrm{m}$ and the ST $0.13 \mu \mathrm{m}$ standard CMOS technologies has been performed. The measurements show that the $0.13 \mu \mathrm{m}$ technology offers a lower breakdown voltage and a lower quenching time. Although both characteristics are desirable in a detector, its dark count rate is higher. In contrast, the $0.35 \mu \mathrm{m}$ technology presents a lower dark count rate and, consequently, a reduced probability of false counts. Indeed, the necessary area to store the false hits is also lower. To have conclusive results, further studies with the dependency of the temperature and sensors irradiated have to be realized. Second, a new pixel with the HV-AMS $0.35 \mu \mathrm{m}$ technology that allows low overvoltage bias operation has been developed. To synchronize the pixel detector with the beam a gated acquisition has been implemented. This mode of operation has allowed us to prove that the dark count rate noise can be reduced in a pixel. By using a front-end circuit based on a floating ground, avalanche detection is possible for very low overvoltages, which also reduces the possibility to have dark counts. The characterization of the fabricated pixel shows that it can provide acceptable performance for tracking systems in future particle colliders.

\section{Acknowledgment}

This work has been partially supported by the National Program for Particle Physics through the Project "Desarrollo de nuevas tecnologías en aceleradores y detectores para los futuros colisionadores de Física de Partículas", coded FPA2008-05979-C04-02.

\section{References}

[1] T. Abe, et al., ILD Concept Group-Linear Collider Collaboration, The International Large Detector: Letter of Intent, arXiv:1006.3396 [hep-ex].

[2] K.D. Stefanov, Nucl. Instr. and Meth. A 549 (2005) 93.

[3] G. Deptuch, G. Claus, C. Colledani, M. Deveaux, A. Gay, W. Dulinski, W. Gornushkin, C. Hu-Guo, M. Winter, Nucl. Instr. and Meth. A 511 (2003) 240.

[4] J.J. Velthuis, R. Kohrs, M. Mathes, A. Raspereza, L. Reuen, L. Andricek, M. Koch Z. Dolezal, P. Fischer, A. Frey, F. Giesen, P. Kodys, C. Kreidl, H. Krüger, P. Lodomez, G. Lutz, H.G. Moser, R.H. Richter, C. Sandow, D. Scheirich, E. von Törne, M. Trimpl, Q. Wei, N. Wermes, Nucl. Instr. and Meth. A 579 (2007) 685

[5] H.G. Moser, Prog. Part. Nucl. Phys. 63 (2009) 186.

[6] D. Pennicard, 3D detectors for synchrotron applications, CERN-Thesis-2009137, University of Glasgow, 2009.

[7] E. Grauges, A. Comerma, L. Garrido, D. Gascon, J. Trenado, A. Diéguez, A. Vilà A. Arbat, L. Freixas, S. Hidalgo, P. Fernández, D. Flores, M. Lozano, Nucl. Instr. and Meth. A 617 (2010) 541.

[8] F. Zappa, S. Tisa, A. Tosi, S. Cova, Sens. Actuators A 140 (2007) 103.

[9] S. Tisa, F. Zappa, A. Tosi, S. Cova, Sens. Actuators A 140 (2007) 113.

[10] C.L. Niclass, Single-photon image sensors in CMOS: picosecond resolution for three-dimensional imaging, PhD Thesis Dissertation 4161, Ecole Polytechnique Fédérale de Lausanne, Switzerland, 2008.

[11] S. Cova, M. Ghioni, A. Lacaita, C. Samori, F. Zappa, Appl. Opt. 35 (1996) 1956. 